PressAcademia Procedia

YEAR 2019 VOLUME 9

Global Business Research Congress, May 30-31, 2019, Istanbul, Turkey.

\title{
PERFORMANCE EVALUATION OF ENSEMBLE LEARNING ALGORITHMS ON UNBALANCED CREDIT SCORING DATA SETS
}

\author{
DOI: 10.17261/Pressacademia.2019.1089 \\ PAP-V.9-2019(36)-p.180-185
}

\section{Nihan Ankara ${ }^{1}$, Hulya Sahinturk ${ }^{2}$}

${ }^{1}$ Yıldız Technical University, Graduate School of Science and Engineering, Mathematical Engineering, Beşiktaş, İstanbul, Turkey nihan.ankara@vakifbank.com.tr, ORCID: 0000-0003-2160-210X

${ }^{2}$ Yıldız Technical University, Mathematical Engineering, Davutpaşa, İstanbul, Turkey hsahin@yildiz.edu.tr, ORCID: 0000-0002-3936-9441

To cite this document

Ankara N., Sahinturk H., (2019). Performance evaluation of ensemble learning algorithms on unbalanced credit scoring data sets. PressAcademia Procedia (PAP), V.9, p.180-185

Permemant link to this document: http://doi.org/10.17261/Pressacademia.2019.1089

Copyright: Published by PressAcademia and limited licenced re-use rights only.

\section{ABSTRACT}

Purpose- As the credit scoring model is developed, the accuracy of the models is low due to the unbalanced distribution of the samples belonging to the classes. In this study, we tried to determine the most effective models by comparing the performance of the models obtained by using collective learning algorithms together with cost sensitive learning method.

Methodology- For this purpose, Bagging and AdaBoost collective learning methods were run on two different credit data sets with decision trees, support vector machines and k-NN basic classifiers. In addition, the penal score of the minority classification group was increased by using cost-sensitive learning method for Bagging and AdaBoost. All these combinations were compared.

Findings- The use of cost-sensitive learning methods has led to more successful results in terms of AUC for both AdaBoost and Bagging. It was observed that the Bagging collective method, which is the main classifier of decision trees, had higher success than the AdaBoost collective method in the case of increasing class imbalance rate in the data.

Conclusion- Although the development of a highly effective credit scoring method is still a problem that needs to be solved, it has been observed that the models created by the collective learning method show higher success than the models created by individual classifiers. This situation coincides with the findings of other studies in the literature. [Maciej Zięba ve ark., 2012]

Keywords: Credit scoring, ensemble learning, unbalance datasets

JEL Codes: C13, C19

\section{DENGESIZ KREDI SKORLAMA VERI SETLERINDE KOLEKTiF ÖĞRENME ALGORITMALARININ PERFORMANS DEĞERLENDIRMESI}

\section{ÖZET}

Amaç- Kredi skorlama modeli geliştirilirken kullanılan veri kümelerinde sınıflara ait örneklerin dengesiz bir dağılıma sahip olmalarından dolayı, modellerin doğruluk oranı düşük olmaktadır. Biz bu çalışmada kollektif öğrenme algoritmalarını maliyete duyarlı öğrenme yöntemiyle birlikte kullanarak elde edilen modellerin performansını karşılaştıp en etkin modellleri belirlemeye çalıştık.

Metodoloji- $\mathrm{Bu}$ amaçla Bagging ve AdaBoost kolektif öğrenme yöntemleri karar ağaçları, destek vektör makineleri ve k-NN temel sınıflandırıcıları ile iki farklı kredi veri seti üzerinde çalıştırılmıştır. Ayrıca Bagging ve AdaBoost için maliyet duyarlı öğrenme yöntemi kullanılarak azınlık sınıflandırma grubunun ceza puanı artırılmıştır. Bütün bu kombinasyonlar kıyaslanmıştır.

Bulgular- Maliyete duyarlı öğrenme yöntemlerinin kullanılması, hem AdaBoost hem de Bagging için performans değerlendirme ölçeği AUC açısından daha başarılı sonuçlar elde edilmesini sağlamıştır. Verideki sınıf dengesizlik oranının artması durumunda, karar ağaçlarının temel sınıflandırıcı olduğu Bagging kolektif yönteminin AdaBoost kolektif yöntemine göre daha yüksek başarı elde ettiği gözlemlenmiştir.

Sonuç- Başarısı yüksek etkili bir kredi skorlama yöntemi geliştirilmesi hala çözülmesi gereken bir problem olmasına rağmen kolektif öğrenme yöntemi ile oluşturulan modellerin bireysel sınıflandırıcılarılarla oluşturulan modellere göre daha yüksek başarı gösterdiği gözlemlenmiştir. Bu durum literatürdeki diğer çalışma bulgularıyla da örtüşmektedir. [Maciej Zięba ve ark., 2012]

Anahtar Kelimler: Kredi skorlama, kolektif öğrenme, dengesiz veri seti

JEL Kodları: C13, C19 


\section{Gíriş}

Son yıllarda artan kredi taleplerine hızlı ve güvenilir yanıtlar vermek finansal kurumlar açısından oldukça önem arz etmektedir. Kredi taleplerinin hızı bir şekilde yanıtlanması, kredi başvurusunda bulunan müşterilerin memnuniyetini; güvenilir bir şekilde yanıtlanması ise finansal kurumların krediyi verdiği müşterisinden geri ödeme noktasında sorun yaşamamasını sağlamaktadır. Finansal kurumların batık kredi oranlarının artması, artan kredi taleplerinin doğru analiz edilmesi ve krediyi geri ödeyen müşteri ve geri ödemeyen müşteri kavramlarının tanımlanabilmesi için kredi skorlama yöntemleri oldukça önemli bir yere sahip olmuştur.

Kredi skorlama, kredi başvurusunda bulunan bireysel veya tüzel kişilerin krediyi geri ödeme noktasındaki yeterliliğini ölçümleyen bir sistemdir. Kredi skorlama sistemi için, müşterinin geçmişteki kredi ilişkilerinde sergilediği davranışlar, gelir-gider dengesi, kredi karşılığında gösterebileceği teminatları ve kefilleri gibi daha birçok puanlamayı etkileyen konular ölçüm için dikkate alınmaktadır.

Günümüzde kredi skorlama sistemlerinde karşımıza çıkan en büyük problemlerden biri verilerin dengesiz dağılmasıdır. Azınlık sınıfın doğru sınıflandırılamaması KÖTÜ müşterilerin belirlenmesi için önerilen modelin tutarlı olmamasına sebep olmaktadır. Sınıf dengesizliği problemini çözmek için çok sayıda yaklaşım önerilmiştir. Bu yaklaşımlar; Ön İşleme, Maliyete Duyarlı Öğrenme, Kolektif Öğrenme’dir.

Dengesiz veri setleriyle başa çıkmak için sınıf dağılımını değiştirerek sınıf dağılımını dengelemek üzere çeşitli ön işleme adımları uygulanmaktadır. Sınıf dağılımı dengeli hale getirilirken Yeniden Örnekleme (Resampling) teknikleri kullanılmaktadır. Yeniden örnekleme, veri örneğini gerçek veri setlerinden istatistiksel olmayan tahmin veya istatistiksel tahmin yoluyla yeniden yapılandırma işlemidir. Yeniden örnekleme teknikleri üç gruba ayrıştırılabilir; [Galar,2012]

$\checkmark \quad$ Random Undersampling: Çoğunluk sınıf örneklerinin rastgele ortadan kaldırılması yoluyla sınıf dağılımını dengelemeyi amaçlayan yeniden örnekleme yöntemidir.

$\checkmark \quad$ Random Oversampling: Azınlık sınıf örneklerinin rastgele çoğaltılması yoluyla sınıf dağılımını dengelemeyi amaçlayan yeniden örnekleme yöntemidir.

$\checkmark \quad$ Synthetic samples: Veriler yetersiz olduğunda orijinal veri setinden yapay olarak üretilmesi yoluyla sınıf dağılımını dengelemeyi amaçlayan yeniden örnekleme yöntemidir. Sentetik veri üretmek için kullanılan yaygın algoritmalar SMOTE ve ADASYN dur.

Maliyete Duyarı Öğrenme, veri sınıflandırmalarında yanlış sınıflandırılmaların maliyetlerinin belirlenmesi ile yanlış sınıflandırmaları dikkate alan bir öğrenme türüdür. [Ling, 2008] Bu öğrenme yöntemi, odaklanılan sınıfın yanlış sınıflandırılması durumundaki ceza puanının artıılarak riski ortadan kaldırmayı hedeflemektedir.

Kolektif öğrenme yöntemi, birden fazla makine öğrenme tekniklerinin aynı problemi çözmek üzere eğitildiği bir makine öğrenme yöntemidir. Bu yöntem, genel sınıflandırma sonuçlarını iyileştirmek için birden fazla sınıflandırıcının sonuçlarını birleştirerek daha güçlü bir sınıflandırıcı elde edilmesini sağlamaktadır.

Kredi skorlama modelinin finansal kurum için en anlamlı çıktısı, kredi geri ödeme durumu ‘KÖTÜ' olarak sınıflandırılmış müşteri kitlesinin önceden tahmin edilebilmesidir. Bu amaçla maliyet duyarlı öğrenme ve kolektif öğrenme teknikleri birleştirilerek yeni bir model önerilmiştir.

\section{LITERATÜR ÖZETi}

Başlıca kolektif öğrenme yöntemleri ve verilerin dengesizliği konusuna yönelik yapılan çalışmalar aşağıdaki gibidir;

$\checkmark \quad$ Verilerin çoğunlukla dengesiz olduğu, bilgilerin eksik olduğu gibi zorluklara sahip IBM İtalyan örneğini temel alan bir vaka çalışması sunulmaktadır. Kredi skorlaması verileri gibi son derece dengesiz veriler için özellikle uygun olan bir kolektif sınıflandırma tekniğinin kullanımı önerilmektedir. (Giuseppe Paleologo ve ark, 2010)

$\checkmark \quad$ Bagging, Boosting ve Stacking kolektif öğrenme yöntemlerinin temel sınıflandırıcıları LR, DT, ANN ve SVM olması durumlarındaki kıyaslamaları incelenmiştir (Gang Wang ve ark, 2011)

$\checkmark \quad$ Beş farklı kolektif öğrenme yönteminin temel sınıflandırıcılarının performansları kıyaslanmıştır. (A.I. Marqués ve ark, 2012)

$\checkmark \quad$ Gürültü verilerinin ve veri gereksiz niteliklerinin etkilerini azaltmak ve nispeten daha yüksek sınıflandırma doğruluğunu elde etmek için Bagging ve Random Subspace kolektif öğrenme stratejisine dayanan çift stratejili kolektif ağaçlar önerilmektedir. (Gang Wang ve ark, 2012)

$\checkmark \quad$ Dengesiz veri dağılımı problemini çözmek üzere (Dynamic Classifier Ensemble Imbalanced Distribution (DCEID)) dinamik bir kolektif sınıflandırıcı yöntemi önerilmektedir. (Jin Xiao ve ark, 2012)

$\checkmark \quad$ Sınıf etiketi tekniklerini değiştirilmesi tekniğinin dengesiz veri kümesinin ortaya çıkması ve asimetrik maliyet matrisi sorunu gibi veri madenciliği problemlerinin çözülmesine yardımcı olduğu incelenmiştir. (Maciej Zięba ve ark, 2012)

$\checkmark \quad$ Kolektif öğrenme yöntemlerinden Bagging ve Boosting yöntemlerinden hangi yöntemin daha performanslı olduğunun araştırılması ve kolektif öğrenme sınıflandırıcılar için bir dizi belirli temel sınıflandırıcının bir araya getirilmesinde, en iyi performansı sağlamak için kaç tane sınıflandırııının kombine edilmesi gerektiği araştırılmıştır. (Chih-Fong Tsai ve ark, 2014)

$\checkmark \quad$ Kolektif sınıflandırıcılar ve hibrit sınıflandırıcılar gibi gelişmiş makine öğrenme tekniklerinin, tek makine öğrenme tabanlı sınıflandırma tekniklerinden daha iyi tahmin performansı sağladığı konularına değinilmiştir. (Tsai Chih-Fong ve ark, 2014)

$\checkmark \quad$ AdaBoost, Bagging, Rotation Forest, Dagging, Decorate ve Random Subspace olmak üzere altı farklı kolektif öğrenme modellerinin performans kıyaslaması yapılmıştır. (Ning Chen ve ark, 2015)

$\checkmark$ Bireysel sınıflandırıcıları, homojen ve heterojen kolektif sınıflandırıcıların kıyaslanması yapılmıştır. (Stefan Lessmann ve ark, 2015)

$\checkmark \quad$ Sınıflandırma doğruluğunu artırmak için yedi ayrı modeli bir araya getiren kolektif bir teknik önerilmiştir. (Shashi Dahiya ve ark, 2015)

$\checkmark \quad$ Büyük dengesiz veriler göz önüne alındığında, kredi skorlama problemleriyle başa çıkabilmek için kolektif öğrenme yönteminde temel sınıflandırıcı olarak LR sınıflandırıcısının kullanılması incelenmiştir. (Hong Wang, Qingsong Xu ve ark, 2015) 
$\checkmark \quad$ AdaBoost, Bagging ve Random Forest olmak üzere üç farklı kolektif öğrenme modellerinin performans kıyaslaması yapılmıştır. (C.R.Durga devi ve ark, 2016)

$\checkmark \quad$ Sınıflar arasındaki dengesizliği azaltmak için başlangıçta dengesiz veri kümesine uygulanan yeni bir yaklaşım sunulmaktadır. (Uma R. Salunkhe ve Suresh N. Mali, 2016)

$\checkmark \quad$ Kolektif öğrenme yöntemlerinin birleşiminin bireysel makine öğrenme yöntemleri ve kolektif öğrenme yöntemlerinden daha iyi performansa sahip olması belirtilmiştir. (You Zhu ve ark, 2017)

\section{VERI VE YÖNTEM}

\subsection{Verilerin Tanıtımı}

Bu çalışmada, UCI Repository veri ambarından 'German Credit' ve 'Australian' veri setleri kullanılmıştır. Veri seti özellikleri arasında müşterilere ait birikim hesaplarının durumu, bankadaki varolan kredileri, aylık geliri gibi finansal ve yaş, medeni durum gibi demografik bilgiler bulunmaktadır.

German Credit Veri Seti; 19 özellik, 1 sınıf etiketi ve 1000 veri içeren orta büyüklükteki eğitim veri seti olarak paylaşılmıştır. Sınıf etiketi değerleri, 'IYY' ve 'KÖTÜ' olarak belirlenmiştir. 'IYi' sınıf etiketi, kredi borcunu geri ödeyen, 'KÖTÜ' sınıf etiketi ise kredi borcunu geri ödemeyen gerçek kişi müşteri kitlesini ifade etmektedir. Eğitim veri setinde sınıf etiketlerine göre veri dağılımları; 'iYi' olarak sınıflandırılan veri sayısı 700, 'KÖTÜ' olarak sınıflandırılan veri sayısı ise 300'dür.

Australian Veri Seti; 14 özellik, 1 sınıf etiketi ve 690 veri içeren kredi kart başvuruları eğitim veri seti olarak paylaşılmıştır. Özelliklerden 6 tanesi nümerik, 8 tanesi ise kategorik veri içermektedir. Veri özelliklerinin açıklamaları belirtilmemiştir. Eğitim veri setinde sınıf etiketlerine göre veri dağılımları; Pozitif sınıf veri sayısı 307, Negatif sınıf veri sayısı ise 383 'tür.

\subsection{Performans Ölçütü}

Makine öğrenme teknikleri model karşılaştırması için Doğruluk Oranı (Accuracy), Duyarlıık (Recall) ve Kesinlik (Precision) ölçülmektedir. Ama son yıllarda kullanılan model başarılarını karşılaştırmak için Alıcı İ̧letim Özellikleri Eğrisi Altında Kalan Alan (AUC-Area Under the Curve) kullanılmaktadır [Fawcett, 2006]

Sistemin başarıııın tek bir değer ile ifade edilmesi ROC eğrisinin altında kalan alan (AUC-Area Under the Curve) ile ifade edilmektedir. Bu alan değeri ne kadar büyük ise sistemin güvenilirlik değeri de o kadar yüksek olur.

\subsection{Kolektif Öğrenme Yöntemleri}

\subsubsection{Bagging}

Her bir modelin bireysel tahminlerinden daha doğru tahminler yapmak üzere çoklu makine algoritmalarının paralel çalışması ile bir araya gelmesini sağlayan bir makine öğrenme yöntemidir. Bu yöntem Leo Breiman tarafından formüle edilmiş ve adı "Boostrap Aggregating" ifadesinin kısaltmasından çıkarılmıştır. [Machovà, 2006] Bagging yönteminin çalışma adımları Şekil 2 de gösterilmiştir.

\subsubsection{Boosting}

Bir dizi zayıf sınıflandırıııyı daha güçlü bir sınıflandıııcıya dönüştüren, her bir iterasyonun sıralı bir şekilde işletilerek daha doğru tahminleme yapıımasını amaçlayan bir makine öğrenme yöntemidir. Bu yöntemde amaç, her bir iterasyonda yanlış sınıflandırılan verilerin ağırlıklarının arttırılması ile bir sonraki iterasyonda yanlış sınıflandırılmış verilere odaklanarak modelin doğruluğunu arttırmaktır. Boosting yöntemlerinin en iyi bilinen algortimaları arasında AdaBoost ve Gradient Boosting algoritmaları yer almaktadır.

\subsubsection{AdaBoost}

1988 yılında Kearns tarafından sorulan bir sorudan ortaya çıkan Boosting yöntemini gerçeklemek üzere 1996 yllında Freund ve Schapire tarafından ilk pratik algoritma olarak AdaBoost algoritması sunulmuştur. [Didrik, 2016] Boosting yönteminin çalışma adımlarında bahsi geçen Adjust_Distribution ve Combine_Outputs ifadeleri gerçek bir algoritmayı ifade etmemektedir. Bu ifadeleri gerçeklemek üzere etkili bir destek algoritması olarak AdaBoost ifade edilmektedir. AdaBoost yönteminin çalışma adımları Şekil 3 te gösterilmiş̧ir.

Şekil 2: Bagging Çalışma Adımları

\begin{tabular}{|l|l|}
\hline \multirow{3}{*}{ GiRDi } & $\begin{array}{l}\text { Veri seti } D=\left\{\left(x_{1}, y_{1}\right),\left(x_{2}, y_{2}\right), \ldots,\left(x_{m}, y_{m}\right)\right\} \\
\text { Temel öğrenme algoritması } \mathcal{L} \\
\text { Öğrenme tur sayısı } T\end{array}$ \\
\hline
\end{tabular}

Şekil 3: AdaBoost Çalışma Adımları

\begin{tabular}{|l|l|}
\hline \multirow{3}{*}{ Girdi } & $\begin{array}{l}\text { Veri seti } D=\left\{\left(x_{1}, y_{1}\right),\left(x_{2}, y_{2}\right), \ldots,\left(x_{m}, y_{m}\right)\right\} \\
\text { Temel öğrenme algoritması } \mathcal{L} \\
\text { Öğrenme tur sayısı } T\end{array}$ \\
\hline
\end{tabular}



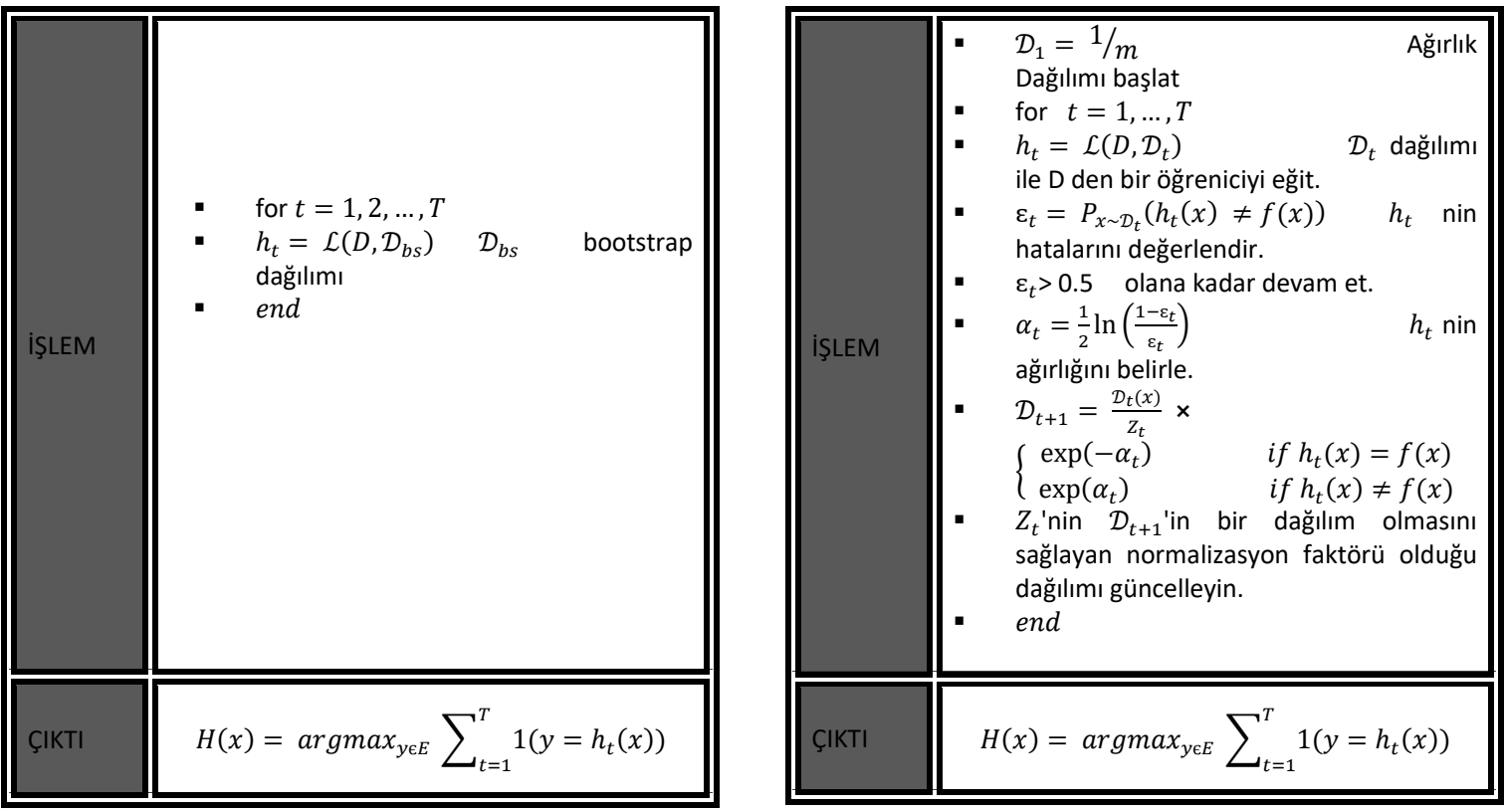

\section{BULGULAR VE TARTISSMALAR}

WEKA programında, 'German Credit' ve 'Australian' veri seti kullanılarak AdaBoost ve Bagging kolektif öğrenme yöntemi REPTree, k-NN ve SVM temel sınıflandırııları ile çalıştırılmıştır. AdaBoost ve Bagging için iterasyon sayıları sırasıyla 257 ve 248 olarak belirlenmiştir. 'German Credit' ve 'Australian'veri setleri için elde edilen sonuçlar;

$\checkmark$ 'German Credit' için doğruluk açısından en yüksek sonuç Bagging-REPTree, en düşük sonuç temel sınıflandırıcı olarak REPTree olmuştur.

$\checkmark$ 'German Credit' için AUC açısından en yüksek sonuç Bagging-SMO, en düşük sonuç AdaBoost-kNN olmuştur.

$\checkmark$ 'Australian' için doğruluk açısından en yüksek sonuç Bagging-kNN, en düşük sonuç AdaBoost-REPTree olmuştur.

$\checkmark$ 'Australian' için AUC açısından en yüksek sonuç Bagging-kNN, en düşük sonuç temel sınıflandırıcı olarak REPTree olmuştur.

WEKA programında, 'German Credit' ve 'Australian' veri seti kullanılarak AdaBoost ve Bagging kolektif öğrenme yöntemi REPTree, kNN ve SVM temel sınıflandırıcıları ile maliyet duyarlı öğrenme yöntemi uygulanarak çalıştırılmıştır. Maliyet duyarlı öğrenme yöntemi 'RiSKLI' müşteri kitlesinin yanlış sınıflandırma ceza puanı diğer sınıfın 2 katı olacak şekilde ayarlanmıştır. AdaBoost ve Bagging için iterasyon sayıları sırasıyla 257 ve 248 olarak belirlenmiştir. 'German Credit' ve 'Australian'veri setleri için elde edilen sonuçlar;

$\checkmark$ 'German Credit' için doğruluk açısından en yüksek sonuç Bagging-REPTree, en düşük sonuç AdaBoost-kNN ve temel sınıflandırıcı olarak kNN olmuştur.

$\checkmark$ 'German Credit' için AUC açısından en yüksek sonuç Bagging-SMO, en düşük sonuç AdaBoost-kNN olmuştur.

$\checkmark$ 'Australian' için doğruluk açısından en yüksek sonuç Bagging-SMO, en düşük sonuç AdaBoost-REPTree olmuştur.

$\checkmark$ 'Australian' için AUC açısından en yüksek sonuç Bagging-kNN, en düşük sonuç temel sınıflandırıcı olarak REPTree olmuştur

'German Credit' ve 'Australian' veri seti için elde edilen deney sonuçlarının maliyet duyarlı öğrenme uygulanıp uygulanmamasına gore doğruluk (accuracy) ve AUC değerleri karşılaştırılmıştır. Karşılaştırmalara göre sonuçlar şu şekildedir;

\section{‘German Credit’ Doğruluk Kriterine Göre Deney Sonuçları}

\begin{tabular}{|c|c|c|c|c|c|c|}
\hline \multirow[t]{2}{*}{ DOĞRULUK } & \multicolumn{3}{|c|}{ MALIYYT DUYARL Ö̈RENME (-) } & \multicolumn{3}{|c|}{$\begin{array}{l}\text { MALIYET DUYARLI OGRENME (+) } \\
\text { (RiskSiz: } 1.0 \text { / RisKLi: 2.0) }\end{array}$} \\
\hline & AdaBoost & Bagging & - & AdaBoost & Bagging & . \\
\hline KNN & 74,2 & 75,2 & 74,2 & 66,8 & 68,8 & 66,8 \\
\hline REPTree & 74,1 & 75,4 & 71,8 & 74,2 & 74,6 & 70,6 \\
\hline SMO & 75 & 75,1 & 75,1 & 72,4 & 72,8 & 72,7 \\
\hline
\end{tabular}

‘Australian' Doğruluk Kriterine Göre Deney Sonuçları

\begin{tabular}{|c|c|c|c|c|c|c|}
\hline \multirow[t]{2}{*}{ DOĞRULUK } & \multicolumn{3}{|c|}{ MALiYET DUYARLI OĞRENME (-) } & \multicolumn{3}{|c|}{$\begin{array}{l}\text { MALiYET DUYARL OC̆́RENME (+) } \\
\text { (Risksiz: 1.0 / RiskLi: 2.0) }\end{array}$} \\
\hline & AdaBoost & Bagging & & AcaBoost & Bagging & \\
\hline KNN & 86,3768 & 86,9565 & 86,3768 & 83,3333 & 83,7681 & 82,029 \\
\hline REPTree & 53,7681 & 54,7826 & 55,0725 & 52,3188 & 55,3623 & 55,5072 \\
\hline SMO & 85,2174 & 85,6522 & 85,6522 & 84,9275 & 85,2174 & 84,7826 \\
\hline
\end{tabular}

\section{‘German Credit’ AUC Kriterine Göre Deney Sonuçları}

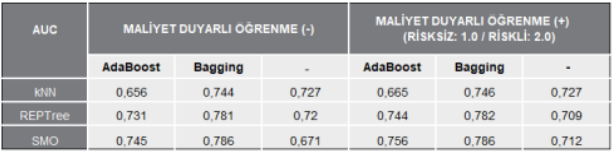

‘Australian AUC Kriterine Göre Deney Sonuçları

\begin{tabular}{|c|c|c|c|c|c|c|}
\hline \multirow[t]{2}{*}{ AUC } & \multicolumn{3}{|c|}{ MALIYYET DUYARL OCGRENME (-) } & \multicolumn{3}{|c|}{$\begin{array}{l}\text { MALiYet DUYARLI OG̈RENME (+) } \\
\text { (RISKSIZ: 1.0/ RiSKLL: 2.0) }\end{array}$} \\
\hline & AdaBoost & Bagging & - & AdaBoost & Bagging & - \\
\hline KNN & 0,876 & 0.918 & 0,915 & 0.879 & 0,919 & 0,872 \\
\hline REPTree & 0.52 & 0.546 & 0.501 & 0.513 & 0.542 & 0.494 \\
\hline smo & 0.88 & 0.908 & 0.863 & 0.885 & 0.897 & 0.845 \\
\hline
\end{tabular}


'German Credit' veri seti için elde edilen deney sonuçlarının maliyet duyarlı öğrenme uygulanıp uygulanmamasına göre 'RISKLI' müşteri tahmin oranı değerleri karşılaştırılmıştır. 'Australian' kredi veri seti için elde edilen deney sonuçlarının maliyet duyarlı öğrenme uygulanıp uygulanmamasına göre 'NEGATiF' tahmin oranı değerleri karşılaştırılmıştır.

'RiSKLi' Müşteri Tahmin Oranı Deney Sonuçları

\begin{tabular}{|c|c|c|c|c|c|c|}
\hline \multirow[t]{2}{*}{$\begin{array}{l}\text { TEMEL } \\
\text { SINIFLANDIRMA }\end{array}$} & \multicolumn{3}{|c|}{ MALiYET DUYARLI OĞRENME (-) } & \multicolumn{3}{|c|}{$\begin{array}{l}\text { MaLiYET DUYARLI OGRENME (+) } \\
\text { (RisKSiz: } 1.0 / \text { RiskL:: 2.0) }\end{array}$} \\
\hline & Adaboost & Bagging & - & AdaBoost & Bagging & - \\
\hline WNN & 0.393 & 0,393 & 0.393 & 0.637 & 0,607 & 0.637 \\
\hline REPTIree & 0,487 & 0,44 & 0,39 & 0,507 & 0.67 & 0.637 \\
\hline Smo & 0.467 & 0.483 & 0.47 & 0.67 & 0.68 & 0.673 \\
\hline
\end{tabular}

'NEGATIF' Tahmin Oranı Deney Sonuçları

\begin{tabular}{|c|c|c|c|c|c|c|}
\hline \multirow[t]{2}{*}{$\begin{array}{l}\text { TTMEL } \\
\text { SINIFLANDIRMA }\end{array}$} & \multicolumn{3}{|c|}{ MALIYET DUYARLI OČRENME (-) } & \multicolumn{3}{|c|}{ 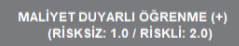 } \\
\hline & AdaBoost & Bagging & - & AdaBoost & Bagging & - \\
\hline KUN & 0,896 & 0,901 & 0,896 & 0,94 & 0.943 & 0,911 \\
\hline REPTIRe & 0.7 & 0.742 & 0,971 & 0.488 & 0,82 & 1 \\
\hline SMo & 0.859 & 0.864 & 0.802 & 0.869 & 0.896 & 0.869 \\
\hline
\end{tabular}

\section{SONUÇ}

Bankacılık sisteminde, kredi verme konusunda müşterilerin belirlenmesinde kredi skorlama modelleri oldukça etkin rol oynamaktadır. Bu amaçla literatürde istatistik ve makine öğrenmesi yöntemleri kullanılarak birçok kredi skorlama modeli geliştirilmiştir. Kredi skorlama modeli geliştirilirken kullanılan veri kümelerinde sınıflara ait örneklerin dengesiz bir dağılıma sahip olmalarından dolayı, modellerin doğruluk oranı düşük olmaktadır. Bu durum kredi skorlama sistemlerinde hala üstesinden gelinmesi gereken bir problem olarak görülmektedir. Bu çalışmada kolektif öğrenme algoritmalarını maliyete duyarlı öğrenme yöntemiyle birlikte kullanarak elde edilen kolektif modellerin performansını karşılaştıp en etkin modellerin belirlenmesine çalışılmıştır. 'RiSKLi' müşteri kitlesinin önceden tahminlenmesindeki başarı oranına odaklanılmıştır.

Bu amaçla Bagging ve AdaBoost kolektif öğrenme yöntemleri karar ağaçları, destek vektör makineleri ve k-NN temel sınıflandırıcıları ile iki farklı kredi veri seti üzerinde çalıştırılmıştır. Ayrıca Bagging ve AdaBoost için maliyet duyarlı öğrenme yöntemi kullanılarak azınlık sınıflandırma grubunun ceza puanı artırılmıştır. Bütün bu kombinasyonlar kıyaslanmıştır.

Bagging kolektif yönteminin AdaBoost kolektif yöntemine göre AUC açısından daha yüksek başarı elde ettiği gözlemlenmiştir.

Maliyete duyarlı öğrenme yöntemlerinin kullanılması German Credit veri setinde, hem AdaBoost hem de Bagging için performans değerlendirme ölçeği AUC açısından daha başarılı sonuçlar elde edilmesini sağlamıştır. Verideki sınıf dengesizliğinin azalması durumunda bu sonuç geçerli olamamıştır.

Çalışmanın odağındaki 'RiSKLi’ müşteri oranı, hem AdaBoost hem de Bagging için temel sınıflandırıcılarla kullanımından daha yüksek orana sahip olmuştur. Maliyet duyarlı öğrenme yönteminin kullanımı bu oranın büyük ölçüde artmasını sağlamıştır. Bu artış veri dengesizliğinin arttığı veri setinde daha büyük oran nispeten veri dengesizliğinin az olduğu durumda daha küçük oranda olmuştur.

Başarısı yüksek etkili bir kredi skorlama yöntemi geliştirilmesi hala çözülmesi gereken bir problem olmasına rağmen kolektif öğrenme yöntemi ile oluşturulan modellerin bireysel sınıflandırıcılarılarla oluşturulan modellere göre daha yüksek başarı gösterdiği gözlemlenmiştir. Bu durum literatürdeki diğer çalışma bulgularıyla da örtüşmektedir. [Maciej Zięba ve ark., 2012]

\section{REFERANSLAR}

Paleologo, Giuseppe ve ark. (2010). Subagging for credit scoring models. European Journal of Operational Research. 201 (2010) $490-499$

Wang, Gang ve ark. (2011). A comparative assessment of ensemble learning for credit scoring, Expert Systems with Applications. 38 (2011) $223-230$

Marqués, A.I. ve ark. (2012). Exploring the behaviour of base classifiers in credit scoring ensembles. Expert Systems with Applications. 39 (2012) 10244-10250

Gang Wang ve ark (2012). A hybrid ensemble approach for enterprise credit risk assessment based on Support Vector Machine. Expert Systems with Applications. 39 (2012) 5325-5331

Jin Xiao ve ark (2012). Dynamic classifier ensemble model for customer classification with imbalanced class distribution. Expert Systems with Applications 39 3668-3675

Maciej Zięba ve ark (2012). Ensemble Classifier for Solving Credit Scoring Problems. IFIP International Federation for Information Processing, pp. 59-66

Chih-Fong Tsai ve ark (2014). A comparative study of classifier ensembles for bankruptcy prediction. Applied Soft Computing 24 (2014) 977984

Tsai Chih-Fong ve ark (2014). Modeling credit scoring using neural network ensembles. Kybernetes, vol. 43, no. 7, pp. 1114-1123

Ning Chen ve ark (2015). Comparative study of classifier ensembles for cost-sensitive credit risk assessment. Intelligent Data Analysis 19 (2015) 127-144

Stefan Lessmann ve ark (2015). Benchmarking state-of-the-art classification algorithms for credit scoring: An update of research. European Journal of Operational Research 247(2015) 124-136 
Shashi Dahiya ve ark (2015). Credit Scoring Using Ensemble of Various Classifiers on Reduced Feature Set. Industrija, vol. 43, no. 4 Hong Wang ve ark (2015). Large Unbalanced Credit Scoring Using Lasso-Logistic Regression Ensemble. PLOS ONE

C.R.Durga devi ve ark (2016). A Relative Evaluation of the Performance of Ensemble Learning in Credit Scoring. IEEE International Conference on Advances in Computer Applications (ICACA)

Uma R. Salunkhe, Suresh N. Mali (2016). Classifier Ensemble Design for Imbalanced Data Classification A Hybrid Approach. International Conference on Computational Modeling and Security (CMS 2016) Procedia Computer Science 85 ( 2016 ) 725 - 732

You Zhu ve ark (2017). Comparison of individual, ensemble and integrated ensemble machine learning methods to predict China's SME credit risk in supply chain finance. Neural Comput \& Applic (2017) 28 (Suppl 1):S41-S50

Mikel Galar ve ark (2012). A Review on Ensembles for the Class Imbalance Problem: Bagging-, Boosting-, and Hybrid-Based Approaches. IEEE Transactions On Systems, Man, And Cybernetics - Part C: Applications And Reviews, vol. 42, no. 4, pp. 463-485

Charles X. Ling, Victor S. Sheng (2008). Cost-Sensitive Learning and the Class Imbalance Problem. Encyclopedia of Machine Learning

Zhou , Zhi-Hua (2012). Ensemble Methods Foundations and Algorithms . Cambridge UK: CRC Press

ETS Asset Management Factory. (2016, Nisan 20). What is the difference between Bagging and Boosting?. Retrieved from https://quantdare.com/what-is-the-difference-between-bagging-and-boosting/

Schapire, R. (2013, Ekim 09). Explaining AdaBoost. Retrieved from http://rob.schapire.net/papers/explaining-adaboost.pdf

Polikar, R. (2008, Aralık 22). Ensemble Learning. Retrieved from http://www.scholarpedia.org/article/Ensemble learning

Lutins, E. (2017, Ağustos 01). Ensemble Methods in Machine Learning: What are They and Why Use Them?. Retrieved from https://towardsdatascience.com/ensemble-methods-in-machine-learning-what-are-they-and-why-use-them-68ec3f9fef5f 